\title{
KERNEL STABLE AND UNIQUELY GENERATED MODULES
}

\author{
SERAP ŞAHINKAYA and TRUONG CONG QUYNH
}

\begin{abstract}
Module theoretic notion of annihilator-stable rings is defined and some characterizations of it are studied in the present paper. $M$ is called a kernelstable module if every element $\alpha \in \operatorname{End}(M)$ satisfies the following condition: if $\alpha(M)+\operatorname{Ker} \beta=M, \beta \in \operatorname{End}(M)$, then $(\alpha-\gamma)(m) \in \operatorname{Ker} \beta$ for an automorphism $\gamma$ of $M$ and for all $m \in M$. For a pseudo-semi-projective module $M$, this notion is equivalent to the uniquely generated module which was defined in [9].
\end{abstract}

MSC 2010. 16U60, 16U99 16E50, 16L30, 19A13

Key words. Stable range, annihilator-stable rings, uniquely generated modules, von Neumann regular rings, unit-regular rings, matrix rings, pseudo-semiprojective module, kernel-stable modules.

\section{REFERENCES}

[1] F.W. Anderson and K. R. Fuller, Rings and categories of modules, Springer-Verlag, New York, 1974.

[2] H. Bass, K-Theory and stable algebra, Publ. Math. Inst. Hautes Études Sci., 22 (1964), $5-70$.

[3] M.J. Canfell, Completion of diagrams by automorphisms and Bass first stable range condition, J. Algebra, 176 (1995), 480-503.

[4] H. Chen, On partially unit-regularity, Kyungpook Math. J., 42 (2002), 13-19.

[5] N.V. Dung, D.V. Huynh, P.F. Smith and R. Wisbauer, Extending modules, Pitman, 1996.

[6] G. Ehrlich, Unit-regular rings, Port. Math., 27 (1968), 209-212.

[7] I. Kaplansky, Elementary divisors and modules, Trans. Amer. Math. Soc., 66 (1949), 464-491.

[8] D. Khurana and T.Y. Lam, Rings with internal cancellation, J. Algebra, 284 (2005), 203-235.

[9] M.T. Kosan, T.C. Quynh and S. Sahinkaya, On rings with associated elements, Comm. Algebra, 45 (2017), 2747-2756.

[10] G. Marks, A criterion for unit-regularity, Acta Math. Hungar., 111 (2006), 311-312.

[11] S.H. Mohammed and B.J. Müller, Continous and discrete modules, London Math. Soc., Vol. 147, Cambridge Univ. Press, 1990.

[12] W.K. Nicholson, Annihilator-stability and unique generation, J. Pure Appl. Algebra, 221 (2017), 2557-2572.

[13] W.K. Nicholson and E. Campos, Morphic modules, Comm. Algebra, 3 (2005), 26292647.

The authors thank the referee for his helpful comments and suggestions.

DOI: $10.24193 /$ mathcluj.2021.1.11 
[14] T.C. Quynh, On pseudo semi-projective modules, Turkish J. Math., 37 (2013), 27-36.

[15] F. Siddique, On two questions of Nicholson, preprint.

[16] J. Von Neumann, Regular ring, Proc. Natl. Acad. Sci. USA, 22 (1936), 707--713.

[17] R. Wisbauer, Foundations of module and ring theory, Gordon and Breach, Reading, 1991.

Received July 22, 2019

Accepted December 3, 2019
Tarsus University

Technology Faculty

Tarsus/Mersin Turkey

E-mail: serap@tarsus.edu.tr

https://orcid.org/0000-0002-2084-6260

Danang University

Department of Mathematics

459 Ton Duc Thang, DaNang city, Vietnam

E-mail: tcquynh@live.com

https: //orcid.org/0000-0002-0845-0175 